tung ist später, wenigstens als Grundlage für eine neue Prüfungsmethode, von Schneider ${ }^{1}$ angefochten worden. In der That ist der Brenzcatechingehalt der Säure meistens zu gering, um bei der ohnehin mehr oder weniger bräınlichen Farbe ihrer ammoniakalischen Lösung die Silborreduction direct deutlich erkennen zu lassen. In allen Füllen lässt sich das Brenzcatechin nachweisen, indem man aus der Benzoësäure das Natronsalz darstellt, dies trockne Salz mit Acther leplacirt und den ätherischon Auszug verdunsten lässt. Die wässrige Lösung des Verdunstungsrückstandes reducirt schon in der Kälte sofort die ammoniakalische Silberlösung und zeigt auch die übrigen charakteristischen Reactionen des Brenzcatechins.

Dies Verfahren ist etwas umständlich und verlangt die Anwendung einer verhältnissmässig grossen Menge Benzoësäure; sonst würde ich dasselbe für jetzt, d. h. so lange kein Fabrikant seine Benzoësäure mit Brenzcatechin versetzt, als die beste Methode zur Unterscheidung der sublimirten Harzbenzoësäure von Benzoësäure andern Ursprungs bezeichnen.

\title{
Ein verbesserter Schwefelwasserstoffapparat.
}

Von Dr. Kubel, Apotheker in Holzminden.

Die Verbesserung des Apparates besteht hauptsächlich in der Aufbewahrung, resp. Conservirung des in dem gebrauchten Apparate zurückbleibenden Schwefeleisens, nach dem Abspülen mit Wasser, unter Glycerin.

In den Apotheken, in denen ein Schwefelwasserstoffapparat nur selten gebraucht wird, ist es wichtig und angenehm, einen Apparat zu haben, der neben andern Vorzügen noch den besitzt, sehr rasch in Thätigkeit, und, nach dem Gebrauche ohne Verlust des ïberschüssigen Schwefeleisens, leicht in einen Zustand versetzt werden zu können, der eine rasche Wiederbenutzung gestattet. Dieser wird erreicht durch Uebergiessen des zurückbleibenden abgespülten Schwefeleisens durch Glycerin. Das Schwefeleisen zeigt unter Glycerin aufbewahrt selbst nach langem Stehen beim Abspülen eine blanke Oberflächo und tritt beim Uebergiessen mit Säure sofort mit dieser in Wechselwirkung.

1) Arch. d. Pharm. 220. S. 408. 
Lässt man dagegen nach dem Gebrauche das abgespülte Schwefeleisen ohne Weiteres in der Flasche zurück, so oxydirt sich dasselbe bekanntlich sehr rasch und wird vollstïndig unbrauchbar, auch lässt sich dic oxydirte Masse meist schlecht ats der Flasche entfernen.

Das Uebergiessen des Schwefeleisens mit Glycerin kann natürlich in jedem Apparate geschehen, in dem dasselbe sich auf dem Boden des Gefüsses befindet, nicht im Pohl'schen, Kipp'schen und einigen anderen.

Es giebt der Schwefelwasserstoffapparate ja eine grosse Menge, verschiedene sind von mir gründlich geprüft, als den zweckmässigsten habe ich den erkannt, der zuerst von Debray zur Entwicklung von Wasserstoff, später von Otto zur Entwicklung von Schwefelwasserstoff empfohlen ist. ${ }^{1}$

Beim täglichen Gebrauche arbeitete der Apparat vorzüglich, dagegen zeigte sich der Uebelstand der raschen Oxydation des zurückbleibenden Schwefeleisens und der damit verbundenen Unannehmlichkeiten in vollem Maasse beim seltenen Gebrauche, wodurch die sonstigen Vorzüge des Apparates sehr beeinträchtigt wurden.

Durch Debergiessen mit Glycerin sind die Uebelstände gänzlich beseitigt, so dass ich den Apparat jetzt als einen in jeder Beziehung vorzüglichen, und auch als einen recht billigen empfehlen kann. Die Anschaffungskosten betragen etwa $3 \mathrm{M}$., seine Zusammenstellung ist eine sehr einfache.

Nachstehend folgt eino Beschreibung des Apparates, wio ich denselben nun schon seit Jahren stets zur Zufriedenheit benutze.

Derselbe besteht aus zwei florentiner Flaschen von je $1 / 8$ Iiter Fassung, ${ }^{2}$ in deren unterer Oeffnung ein Kork mit kurzem Glasrohr befestigt wird. Die obere Oeffnung der Entwicklungsflasche wird mit einem Korke verschlossen, durch den ein oberhalb knieförmig gebogenes Glasrohr geht, welches durch ein Gummirohr mit einem gleich gebogenen Glasrohre verbunden ist, dessen längerer Schenkel durch den Kork einer zweckmässigen Waschflasche von Stender (Höhe 18 Centim.) bis auf den Boden reicht. In der seitlichen Oeffnung der Waschflasche ist ebenfalls ein zweischenkliches Glas-

1) Graham-Otto's Iehrbuch. 4. Aufl. I. Abth. S. 615.

2) von Stender Glashütte bei Lamspringe à $75 \mathrm{Pf}$. 
376

Kubel, Verbesserter Schwefelwasserstoffapparat.

rohr befestigt, an dem mit Hülfe eines Gummirohres das gerade, ziemlich enge Glasrohr befestigt wird, welches das Gas direct in die zu behandelnde Flüssigkeit leitet.

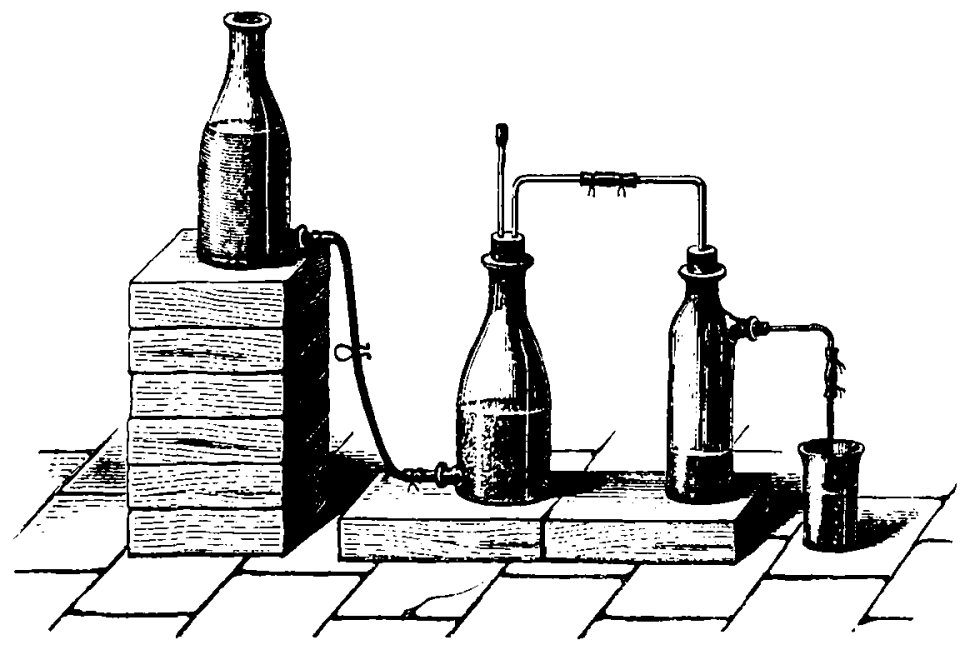

Die beiden Hauptflaschen sind durch einen etwa 20 Centim. langen Gummischlauch, der durch einen Quetschhalm geschlossen werden kann, verbunden. In die Entwicklungsflasche kommt zuerst eine Schicht bohnengrosser Kiesstückchen, die besser wie Kohlenstückchen sind, darauf Scliwefeleisen in ähnlich grossen Stücken, bis die Flasche nahezu zur Hälfte gefüllt ist, ausreichend für einen längeren Gebrauch, in die Waschflasche etwas Wasser und dann bei geschlossenem Quetschhahne in die oben offene Säureflasche mehr oder weniger verdünnte Salzsäure, etwa 1 Thl. Säure, 2 Theile Wasser, je nachdem längere oder kürzere Zeit Gas entwickelt werden soll. Diese Flasche wird von vornherein höher gestellt, wie die Entwicklungsflasche. Nach dem Oeffnen des Quetschhahnes fliesst die Säure zu dem Schwefeleisen, und die Gasentwicklung beginnt. Man hat es ganz in der Hand, durch höher oder niedriger Stellen der Säureflasche, resp. durch mehr oder weniger starkes Oeffnen des Quetschhahnes (durch Schraube oder zwischengeklemmtes Holzstïckchen) die Gasentwicklung zu verstärken oder zu mässigen, so dass bei einiger Aufmerksamkeit leicht stundenlang eine ziemlich gleichmässige Gasentwicklung zu erzielen ist; man hat nur nöthig, den Rand der Säureflasche oder des Quetschhahnes nach Bedürfniss etwas 
zu ändern und den Inhalt der ersteren ab und zu umzuschwenken, um die etwa zurückgetretene concentrirte Salzlösung wieder mit der Säure zu mischen.

Ist die Säure gänzlich verbraucht, so stellt man die Säureflasche so tief, dass alle Flüssigkeit aus der Entwicklungsflasche in jone fliesst, wobei die Verbindung zwischen letzterer und Waschflasche unterbrochen oder ein in dem Korke der Entwicklungsflasche befindliches, für gewöhnlich durch Gummikappe verschlossenes Luftröhrchen geöffnet wird, lässt die unbrauchbare Flüssigkeit fortlaufen, füllt neue Säure in die Flasche, und die Gasentwicklung beginnt von neuem.

Wird die Entwicklung unterbrochen, ehe alle. Säure verbraucht und soll dieselbe aufbewahrt werden, so lässt man alle Flüssigkeit in die Säureflasche zurücksteigen, schliesst dann die obere Oeffnung derselben mit einem Korke, legt die Flasche auf die Seite, dass die seitliche Oeffnung sich obenauf befindet, entfernt Kork mit Glasrohr und vorschliesst die Oeffnung mit einem anderen Korke. Soll der Apparat nicht weiter benutzt werden, so lässt man, wenn sich die Aufbewahrung überschüssiger Säure nicht mehr lohnt, alle Flüssigkeit ablaufen, nimmt don Apparat auseinander, spült alle Theile mit Wasser $a b$, schliesst die seitliche Oeffnung der Entwicklungsflasche mit einem Korke und übergiesst das zurückgebliebene Schwefelcisen mit Glycerin. Bei der Wiederbenutzung lässt man das Glycerin ablaufen, dasselbe wird wiederholt benutzt, spült den Rest mit Wasser ab, setzt den Apparat, wie angegeben zusammen und sofort beginnt bei Zutritt der Säure die Gasentwicklung.

In dem Falle, wo Spuren von Arsen in den Materialien zur Schwefelwasserstoffentwicklung $\mathrm{zu}$ vermeiden sind, statt Schwefeleisen Schwefelcalcium oder Schwefelbaryum benutzt werden muss, ist der besprochene Apparat ebenfalls sehr gut zu gebrauchen.

Man schüttet das Schwefolmetall (ohne Kiesstückchen) in die Entwicklungsflasche, feuchtet mit etwas Wasser an und lässt die reine Säure nach und nach zutreten, so dass eine gleichmässige Gasentwicklung stattfindet. Ist das Schwefelmetall zersetzt, so lässt man alle Flüssigkeit zurücktreten, schüttet eine neue Menge Schwefelmetall in die Entwicklungsflasche u. s. w. Der Inhalt der letzteren ist $\mathrm{ab}$ und zu aufzurütteln.

Soll gelegentlich cine kleine Menge Schwefelwasserstoffgas in eine Flüssigkeit geleitet werden, so lässt sich zweckmässig der kleine 
Handapparat benutzen, wie ihn Otto l. .c S. 614 beschrieben hat (Kochflasche mit gebogenem Glasrohre); auch in diesem Apparato wird nach dem Gebrauche das abgespülte Schwefeleisen mit Glycerin übergossen. Nach dem Abgiessen desselben ist der Apparat sofort wieder brauchbar.

\section{B. Monatsbericht.}

\section{Nahrungs - und Genussmittel, Gesundheitspflege.}

Luft. - B. Fischer und B. Proskauer stellten umfangreiche Versuche an über die desinficirende Kraft des Chlors und des Broms. Dieselben waren durch den Umstand veranlasst worden, dass die zur Desinfection geschlossener Räume empfohlene schweflige Säure durchaus nicht den Anforderungen entspricht, welche man an ein moderne Desinfectionsmittel $\mathrm{zu}$ stellen hat.

Chlor. - Nach den Versuchen der Verf. ist von ihnen kein Mikroorganismus gefunden worden, dessen Tödtung dureh Chlor nicht gelungen wäre. Alle Mikroorganismen, und zwar in allen ihren Lebenszuständen, können daher mit Hilfe von Chlor vernichtet werden. Bei diesen Desinfectionen durch Chlor spielt die vorhandene Feuchtigkeit eine hervorragende Rolle. Ist die Luft mit Feuchtigkeit gesättigt, so lässt sich annehmen, dass eine sichere Desinfection aller in lufttrockenem Zustande befindlichen Mikroorganismen, vorausgesetzt, dass sie nicht in zu dicker Schicht angeordnet und nicht besonders umhüllt sind, erreicht wird, wenn ein Chlorgehalt von 0,3 Vol. Proc. 3 Stunden lang, resp. ein solcher von 0,04 Vol. Proc. 24 Stunden lang einwirkt. Diese zunächst nur in Glasflaschen ausgeführten Versuche fanden auch eine theilweise Bestätigung bei der Desinfection eines Kellerraumes. Die Wirksamkeit des Chlors würde sich hierbei vermuthlich noch haben steigern lassen, wenn schon längere Zeit vor der eigentlichen Desinfection die Luft des betreffenden Raumes auf einen möglichst hohen Grad von Feuchtigkeit gebracht und auf einem solchen erhalten worden wäre. Die in dem Kellerraume ausgeführten Versuche zeigten, dass bei Verwendung von Chlor zwar keine absolut zuverlässige Desinfectionsleistung erzielt wird, jedoch Resultate erreicht werden, die im Vergleich mit anderen Desinfectionsmitteln als recht bedeutende $\mathrm{zu}$ bezeichnen sind, insofern als man dabei auf die Vernichtung des grössten Theiles der oberflächlich gelegenen Infectionskeime, selbst auch der widerstandsfähigsten, rechnen kann. Unter allen Umständen muss man darauf dringen, dass sobald sich Jemand entschlossen hat, einen Raum mit Hülfe eines gasförmigen Mittels zu desinficiren, er dem Chlor vor der sehwefligen Säure den Vorzug giebt. Soll nun das Chlor hierzu benutzt werden, so darf man nicht unter eine Concentration von 0,3 Vol. Proc. heruntergehen. Von den verschiedenen Verfahren der Chlorentwicklung verdient die Entwicklung aus Chlorkalk und Salzsäure zu obigen Zwecken entschieden den Vorzug, da hierbei eine vollständige Zersetzung ohne Anwendung von Wärme stattfindet. Die hierdurch bedingten Kosten belaufen sich anf circa 15 Pfennig pro Kubikmeter. Für die Praxis dürfte es sich empfehlen, in einem Gefässe nie mehr als 0,5 Kilo 